\title{
USO DE FÉRULAS CORRECTORAS EN LA RIGIDEZ DE LA ARTICULACIÓN INTERFALÁNGICA PROXIMAL COMO CONSECUENCIA DE UN TRAUMATISMO
}

\author{
CORRECTIVE SPLINTS IN THE STIFFNESS OF THE PROXIMAL INTERPHALANGEAL \\ JOINT AS A RESULT OF TRAUMA
}

\section{Daniel Gan Benedí ${ }^{*}$}

\begin{abstract}
Resumen
La pérdida de rango de movimiento pasivo (PROM) en las articulaciones interfalángicas de la mano es una secuela común tras lesiones traumáticas. A pesar de que el uso de férulas se ha convertido en una modalidad terapéutica ampliamente aceptada para mejorar el PROM, existe muy poca evidencia empírica que guíe en la prescripción de férulas. El presente estudio investiga el efecto del uso de férulas correctoras en contracturas en flexión de la articulación interfalángica proximal (IFP) de los dedos de la mano tras una lesión traumática. Diecisiete pacientes con una contractura en flexión de la IFP tras una lesión traumática como consecuencia de un accidente laboral participaron en este estudio experimental. Mediante un goniómetro digital se valoró el PROM de la articulación IFP de dichos pacientes y se les asignó de manera aleatoria a uno de los dos grupos de férulas. A los pacientes del grupo A se les colocó una férula de extensión dinámica de la IFP y a los del grupo B se les colocó una férula de extensión estática. El PROM de la articulación se volvió a valorar con un goniómetro en el momento en el que los pacientes recibieron el alta laboral. Los resultados indicaron mejorías significativas en ambos grupos en la corrección de la contractura en flexión $(p<0,05)$. La diferencia entre los pacientes que usaron la férula dinámica frente a los que usaron la férula estática no fue estadísticamente significativa. Ambos tipos de férulas pueden ser recomendadas para el tratamiento de contracturas en flexión inferiores a $35^{\circ}$ de la IFP tras una lesión traumática.
\end{abstract}

\section{Palabras clave:}

Articulación interfalángica proximal; Contractura en flexión; Rehabilitación; Férula dinámica; Férula estática

\begin{abstract}
Reduced passive range of motion (PROM) of the interphalangeal joints of the hand is a common consequence after a traumatic injury. Although mobilizing splinting has become a rehabilitative approach widely accepted by therapists to improve PROM, limited empirical evidence is available to guide in its prescription. This study investigates the effect of corrective splinting on flexion contracture of the finger proximal interphalangeal joints (PIP) after a traumatic injury of the hand. Seventeen patients with finger flexion contracture of the PIP as a result of an industrial accident participated in the experimental study. The passive range of motion of the PIP joint was measured with a finger goniometer and patients were randomly allocated to one of two splint programs. Subjects in group A were given dynamic extension splints, and subjects in group B were given static splints. PROM was re- assessed with a goniometer once the patient was discharged and started working again. Results indicated significant improvement in both groups in the correction of the finger flexion contracture $(p<0,05)$. Patients with dynamic finger extension splints did not differ significantly from those with static splints in extension gains. Both types of splints can be recommended for flexion contractures of 35 degrees or less in the PIP joint after a traumatic injury.
\end{abstract}

\section{Keywords:}

Proximal interphalangeal Joint; Flexion contracture; Rehabilitation; Dinamic split; Static splint

1 * Terapeuta Ocupacional. Profesor asociado del Grado de Terapia Ocupacional en la Facultad de Ciencias de la Salud de Zaragoza. Universidad de Zaragoza (España).

CORRESPONDENCIA: Daniel Gan Benedí. Universidad de Zaragoza-Facultad Ciencias de la Salud. Departamento de Fisiatria y Enfermería. C/ Domingo Miral s/n. C.P. 50009. Zaragoza (España) Correo electrónico: dgan@unizar.es 


\section{INTRODUCCIÓN}

La articulación interfalángica proximal (IFP) y su movimiento resultan claves para la funcionalidad de la mano (Prosser, 1996). Como resultado de la inflamación e inmovilización tras un traumatismo, se puede desarrollar una contractura en flexión de dicha articulación. Si la articulación IFP no se puede extender totalmente, entonces el agarre está limitado.

Con relativa frecuencia, el clínico se enfrenta a problemas en la restauración del rango de movimiento en las articulaciones de la mano tras lesiones traumáticas en la extremidad superior (Frost HM, 1990), siendo aceptado como un método eficaz el uso de férulas de mano para la mejora del rango de movimiento (ROM), mediante la aplicación de una tensión suave prolongada en presencia de rigidez articular. (Callahan, 1986).

Esta práctica se apoya en la observación de Brand (1995) de que "toda elongación del tejido lograda por el estiramiento se acortará de nuevo cuando la fuerza esté relajada" y por los hallazgos similares de Frank et al. (1985) de que "Ios ligamentos volverán a la longitud de pre-estiramiento". Se sabe que la rigidez de las articulaciones resulta del período de inmovilización tras un traumatismo (Peacock EE.,1983), siendo el principal tejido implicado en la rigidez articular el tejido conectivo periarticular (Akeson, 1980).

En 1976, Arem y Madden (1976) demostraron el alargamiento del tejido cicatrizal cuando éste era sometido a un estrés prolongado y suave y Light et al. (1984) informaron que una carga suave, prolongada era más eficaz que el estiramiento breve aplicando una gran tensión en el tratamiento de las rodillas rígidas en seres humanos.

Años más tarde, Flowers y LaStayo (1994) postularon que el aumento en el rango de movilidad era proporcional a la longitud de tiempo que la articulación se mantenía en la posición de máxima elongación, inspiración para el concepto TERT (tiempo total en máxima elongación, calculado multiplicando la frecuencia y la duración del tiempo en que la articulación se mantiene en el máximo rango tolerable). El TERT cuantifica la dosis de tratamiento recibido por el paciente, en un momento dado (Wilton J. Hand Splinting, 1997).

Así pues, el aumento del rango de movimiento pasivo (PROM) en la rigidez de las articulaciones es directamente proporcional al tiempo que la articulación se mantiene en el rango final pero la intensidad de la fuerza aplicada por lo general está limitada por la tolerancia al dolor del paciente. En férulas estáticas, la intensidad puede ser ajustada mediante la tensión que ejerce el velcro sobre la articulación o variando el ángulo de la férula. En férulas dinámicas la intensidad se puede ajustar modificando la tensión en el componente elástico del dispositivo. En cualquier caso, el restablecimiento de la normalidad en la longitud de un tejido que se ha acortado después de desuso, exige mantener el tejido en una moderada posición elongada durante un tiempo significativo que favorezca su crecimiento.

Otros factores que se sabe limitan la cantidad y duración de la aplicación de la fuerza, además de la tolerancia al dolor individual, son la circulación local, la tolerancia de la piel a la presión o la etapa de reparación de los tejidos. Glasgow et al. (2003) concluyeron que el tiempo total diario adecuado necesario para una resolución óptima de una contractura articular se situaba entre 6 y 12 horas.

A pesar del uso frecuente de férulas para mejorar el PROM, existe escasa evidencia empírica que guíe al clínico en la prescripción de férulas y el tiempo de llevarlas. Una guía útil para el razonamiento clínico en la prescripción y la aplicación de férulas ha sido proporcionada por McClure et al. (1994). Estos autores describen un algoritmo que tiene en cuenta los parámetros de la intensidad de la fuerza de la férula, intervalo de tiempo total final, dolor y el progreso del ROM y requiere de una modificación continua según la respuesta del paciente al tratamiento. El objetivo es que este algoritmo pueda servir de guía en las decisiones de los terapeutas en el uso de férulas para el tratamiento de pacientes con ROM limitado.

Flowers (2002), plantea una guía de toma de decisiones, basada en la evaluación clínica del estiramiento de los tejidos, para la planificación de un programa de empleo de férulas en pacientes con rigidez articular. Sugiere medir el rango de movimiento antes y después de un programa de acondicionamiento previo que incluye 10 minutos de calor y ejercicio. Ganancias en el ROM de al menos $20^{\circ}$ sugieren continuar con ejercicios de movimiento activo y no usar férulas. Si la ganancia es de $15^{\circ}$, considera la articulación ligeramente rígida y entonces recomienda el uso de férulas estáticas. Si la ganancia es aproximadamente de $10^{\circ}$, la articulación es significativamente rígida y necesita de una sobrepresión para aumentar la tensión, requiriendo esta articulación probablemente una férula dinámica. Ganancias del orden de $5^{\circ}$ o menos, indican que la articulación es muy rígida y requiere de una intervención más agresiva (uso de una férula estática progresiva que ejerza gran presión). Cabe recordar, no obstante, que 
la mayoría de las recomendaciones sobre el protocolo más adecuado en la aplicación de férulas se basan únicamente en la experiencia clínica.

La eficacia en el uso de férulas durante la etapa de recuperación desde fases tempranas está más que demostrada. (Flowers K., 2002;. Michlovitz SL., Harris BA., Watkins MP, 2004; Glasgow C., Fleming J., Tooth L., 2008; Fess EE., McCollum M., 1998; Abbiati G., Delaria G., Saporiti E., Petrolati M., Tremolada C.,1995; Freeland AE., Hardy MA., Singletary S., 2003).

Sin embargo, son pocos los estudios que informan de si el hecho de usar una férula estática o dinámica influye en la corrección de deformidades de los dedos, habiéndose realizado la mayoría en contracturas articulares de origen traumático y bajo un análisis teórico más que con ensayos clínicos. Estudios como el de Fairleigh y Hacking (1988) sobre la aplicación de férulas dinámicas en pacientes sometidos a artroplastia o el de Palchik et al. (1990) sobre la aplicación de férulas dinámicas en el manejo de la deformidad de Boutonniere, han documentado que las férulas dinámicas de tracción son eficaces en la prevención y corrección de deformidades de los dedos. Sin embargo, Wu (1990) encontró más eficaz el uso de férulas estáticas no elásticas correctivas para la contractura en flexión de la articulación IFP, mientras que Li (1999), tras un análisis biomecánico de férulas digitales, afirmó que la férula dinámica tipo Capener era más eficaz que la estática (belly gutter) para la corrección de la contractura en flexión de la articulación IFP.

Tradicionalmente el diseño de la férula se realiza sobre la base de la experiencia clínica del terapeuta en el tratamiento de casos similares. La literatura aún no proporciona pautas objetivas para determinar la férula de elección.

Desde el punto de vista biomecánico, parece clara la inconveniencia de usar férulas estáticas comerciales basadas en el sistema de tres puntos de apoyo en contracturas en flexión mayores de $35^{\circ}$. Sin embargo, la evidencia que avale el tipo de férula más conveniente en el tratamiento de contracturas en flexión menores de $35^{\circ}$ es escasa.

\section{Objetivos}

El objetivo de este ensayo clínico es examinar los efectos de la aplicación de férulas correctoras en contracturas en flexión de los dedos tras una lesión traumática y comparar los resultados de dos tipos diferentes de férulas correctoras (estática y dinámica) en el rango de movilidad de la articulación IFP en contracturas en flexión inferiores a $35^{\circ}$.

\section{Material y Métodos}

\section{Pacientes}

Se trata de un ensayo clínico aleatorio prospectivo que incluye trabajadores de entre 18 y 65 años que sufrieron un accidente laboral y acudieron al Hospital Mutua de Accidentes de Zaragoza de España (HMAZ). Los pacientes seleccionados debían cumplir los siguientes criterios: haber sido vistos por un médico traumatólogo y un médico rehabilitador; presentar una lesión traumática por fractura cerrada de falange(s) de la mano, herida abierta de dedo(s) de la mano con afectación ligamentosa y/o luxación interfalángica por sobreesfuerzo físico; presentar como secuela del accidente una pérdida del ROM activo y pasivo en la articulación IFP del II, III, IV o V dedo de la mano; haber sido prescrito el uso de una férula de extensión para la articulación IFP; ser derivado al Servicio de Terapia Ocupacional del HMAZ y haber recibido tratamiento rehabilitador.

Los pacientes fueron excluidos sólo fueron atendidos en urgencias, no vistos por un especialista en traumatología y un especialista en rehabilitación, presentaban afectación de más de un dedo y/o mano/antebrazo, no realizaban el tratamiento rehabilitador en el HMAZ, si el motivo de la lesión difería de los establecidos en los criterios de inclusión o presentaban afectación del sistema nervioso central, condiciones inflamatorias o prótesis articulares.

\section{Procedimiento}

En la visita inicial todos los pacientes fueron informados del estudio. Todos los sujetos incluidos en el estudio dieron su consentimiento para la participación en el mismo. El estudio fue aprobado por el Comité de Ética del HMAZ.

Aquellos pacientes con una contractura en flexión menor de $35^{\circ}$ fueron asignados aleatoriamente a uno de los dos grupos de férulas, estática y dinámica. Se prepararon dos sobres idénticos con una tarjeta en su interior para el primer paciente. Una tarjeta contenía la palabra dinámica (Grupo A) y el otro la palabra estática 
(Grupo B). El primer paciente derivado al Departamento de Terapia Ocupacional eligió un sobre y al siguiente sujeto seleccionado se le asignó el sobre que quedaba. Se realizó este procedimiento con todos los pacientes que fueron derivados al Departamento para conseguir un número similar de sujetos en ambos grupos.

$\mathrm{Si}$ el sujeto presentaba una contractura en flexión mayor de $35^{\circ}$ no se le asignaba a ningún grupo y, siguiendo las recomendaciones biomecánicas existentes en la literatura, (Fess, 1988; Fess, 1987; Colditz J.,2000) se le colocaba una férula dinámica.

El mismo terapeuta que llevó a cabo las evaluaciones del rango articular antes y después del tratamiento colocó la férula correspondiente. Los sujetos incluidos en cada grupo no fueron informados de las características de la férula del grupo alternativo.

Tras la inmovilización del dedo por el accidente, el déficit en extensión se intentó corregir mediante rehabilitación y una férula de extensión estática o dinámica, llevada unas horas durante el día y toda la noche en aquellos pacientes que lo tolerasen hasta que la extensión activa se recuperó.

Los datos recogidos fueron: edad, sexo, número de sesiones de rehabilitación, tiempo de uso de la férula, diagnóstico, y los días de seguimiento hasta la fecha del alta.

Todos los participantes recibieron el mismo tratamiento rehabilitador de movilizaciones pasivas y ejercicio activo. Recibieron un total de 5 sesiones semanales de fisioterapia y terapia ocupacional a lo largo del proceso de rehabilitación en el HMAZ. Se animó a los sujetos a usar la mano lesionada en actividades funcionales cuando no llevasen la férula.

\section{Selección de las férulas}

Ya sean prefabricadas o realizadas a medida, los diseños existentes de férulas para la movilización en extensión de la articulación IFP son numerosos (Colditz J., 2000). Para este trabajo se decidió utilizar dos férulas disponibles comercialmente con las que el terapeuta estaba familiarizado. A pesar de las posibles dificultades de ajuste en el paciente, la decisión de utilizar férulas prefabricadas respondió a la necesidad de establecer condiciones lo más similares posibles entre los sujetos de la investigación, evitando que el estudio se viera influido por las modificaciones en el diseño, además de facilitar la posibilidad de repetir dichas condiciones en estudios posteriores. Hunter.
Siguiendo las recomendaciones de Glasgow et al. (2003) se explicó a los sujetos que debían llevar la ortesis entre 6 y 12 horas diarias según la tolerancia de cada paciente. Inicialmente se estableció la intensidad de tal manera que el paciente pudiera usar la férula sin aumento del dolor durante al menos 20 minutos. El uso de las férulas podía ser intermitente o continuo, según variables individuales en cuanto a la circulación y la tolerancia al dolor. Las fuerzas aplicadas por la férula debían ser tolerables durante un período prolongado de tiempo para efectuar un cambio en la formación de colágeno.

Los pacientes del grupo A recibieron una férula dinámica tipo Bort con una muy buena tolerancia parte del paciente (Imagen 1). Cada paciente del grupo B recibió una férula de extensión de los dedos estática tipo Safety Pin (Imagen 2).

\section{Imagen 1 \\ Férula dinámica Bort Mobidig ${ }^{\circledast}$}

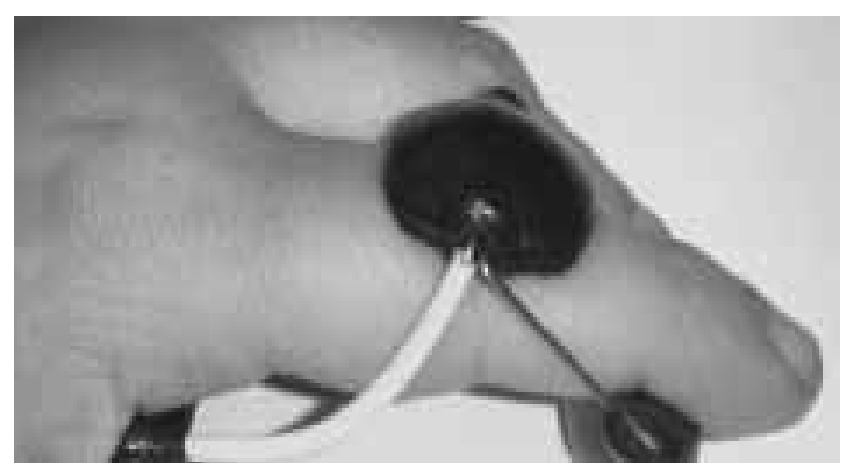

Imagen 2

Férula estática tipo Safety Pin

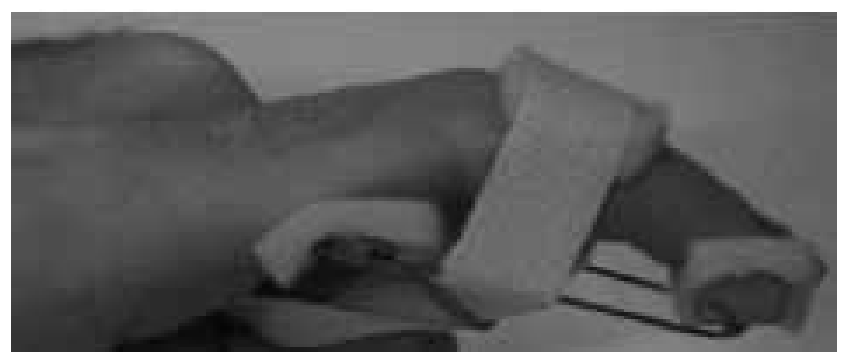

El terapeuta explicó el mecanismo de las férulas y los procedimientos de uso a todos los pacientes. Si sentían algún dolor o molestia en la articulación después de usar la férula, debían informar de ello. 


\section{Medidas de resultado}

La valoración de la efectividad del uso de las férulas se basó en el aumento del rango de movimiento articular. Un goniómetro estándar digital, cuya aplicabilidad ha sido validada en la articulación IFP (Fess, 2004; Hunter, 1995) fue utilizado para la valoración del PROM de la articulación IFP. El preacondicionamiento de los tejidos periarticulares antes de medir el PROM resulta un intento de estandarizar las condiciones para la medida, haciéndolas más fiables y significativas. En nuestro estudio consistió en diversas formas de ejercicios activos y pasivos y movilización de las articulaciones.

\section{Análisis de los datos}

En aquellos sujetos con una contractura en flexión inicial mayor de $35^{\circ}$ se analizaron los datos del rango articular antes y después del tratamiento con férulas para determinar si hubo cambios en el PROM mediante la prueba t-student para muestras relacionadas.

En aquellos sujetos con una contractura en flexión inicial menor de $35^{\circ}$ se realizó, en primer lugar, una prueba t-student para muestras relacionadas para determinar si hubo diferencias antes y después de la intervención, sin tener en cuenta el diseño de la férula y en segundo lugar, un t-test para muestras independientes para determinar si existió diferencia en el rango articular entre las dos férulas empleadas.

Para el análisis de los datos se utilizó el programa informático de estadística SPSS versión 17. El nivel de significación se situó en $\mathrm{p}<0.05$.

\section{Resultados}

Se colocaron férulas de extensión de la articulación IFP a un total de 31 pacientes en el Departamento de Terapia Ocupacional del HMAZ. Todos los pacientes eran trabajadores que habían sufrido una lesión traumática como consecuencia de un accidente laboral. De todos ellos, un total de 17 voluntarios (5 mujeres y 12 hombres) mayores de edad que cumplían los criterios específicos de selección fueron reclutados para el estudio. Todos los sujetos seleccionados tenían antecedentes de lesión traumática en la mano seguida de una reducción en el rango de movimiento. Las características de la muestra se indican en la tabla 1.

Se asumió que la limitación de la extensión de los pacientes seleccionados se debió a la inactividad prolongada del aparato extensor.

Tras colocarles la férula, los 17 pacientes seleccionados fueron evaluados de nuevo en el momento del alta. El seguimiento medio fue de 72,82 días (rango 22174). Recibieron una media de 35 sesiones de rehabilitación y llevaron una media de 73 días la férula.

Todos los participantes completaron el estudio. Los grupos no mostraron diferencias significativas en el grado de contractura en flexión de los dedos afectados. El grado promedio inicial de la contractura en flexión fue de $33,12^{\circ} \pm 11,6^{\circ}$ entre los 17 participantes.

Tabla 1

\section{Características de los participantes}

\begin{tabular}{|c|c|c|c|}
\hline & $\begin{array}{c}\text { Férula de extensión } \\
\text { dinámica }>35^{\circ} .\end{array}$ & $\begin{array}{c}\text { Férula de extensión dinámica } \\
<35^{\circ}(\text { Grupo }) .\end{array}$ & $\begin{array}{c}\text { Férula de extensión estática } \\
\text { (Grupo B). }\end{array}$ \\
\hline Pacientes & 5 & 6 & 6 \\
\hline Hombre/mujer & $4 / 1$ & $3 / 3$ & $5 / 1$ \\
\hline Edad media & 46,20 & 47,5 & 36 \\
\hline Dedo afectado & & & 0 \\
II & 2 & 0 & 1 \\
III & 2 & 2 & 1 \\
IV & 0 & 1 & 4 \\
V V & 1 & 3 & \\
\hline
\end{tabular}




\begin{tabular}{|c|c|c|c|}
\hline & $\begin{array}{c}\text { Férula de extensión } \\
\text { dinámica }>35^{\circ} .\end{array}$ & $\begin{array}{c}\text { Férula de extensión dinámica } \\
<35^{\circ} \text { (Grupo A). }\end{array}$ & $\begin{array}{c}\text { Férula de extensión estática } \\
(\mathrm{Grupo} B) .\end{array}$ \\
\hline $\begin{array}{c}\text { Tipo de lesión } \\
\text { Corte con afectación de } \\
\text { tendón }\end{array}$ & 2 & 1 & 2 \\
Fractura & 3 & 4 & 3 \\
Luxación & 0 & 1 & 1 \\
\hline
\end{tabular}

Los resultados (Gráficos 1 y 2 ) mostraron mejoras significativas para todos los pacientes en la corrección de la contractura en flexión de la articulación IFP, independientemente del grado de contractura inicial o del tipo de férula utilizada. Ello indica que un programa de intervención combinado de movilización pasiva, ejercicios activos y férulas correctivas resulta eficaz en la corrección de la contractura en flexión de la articulación IFP.

\section{Gráfico 1}

Valores de media de los grados de extensión de la IFP antes y después de la intervención con férulas en pacientes con contractura mayor de $35^{\circ}$.

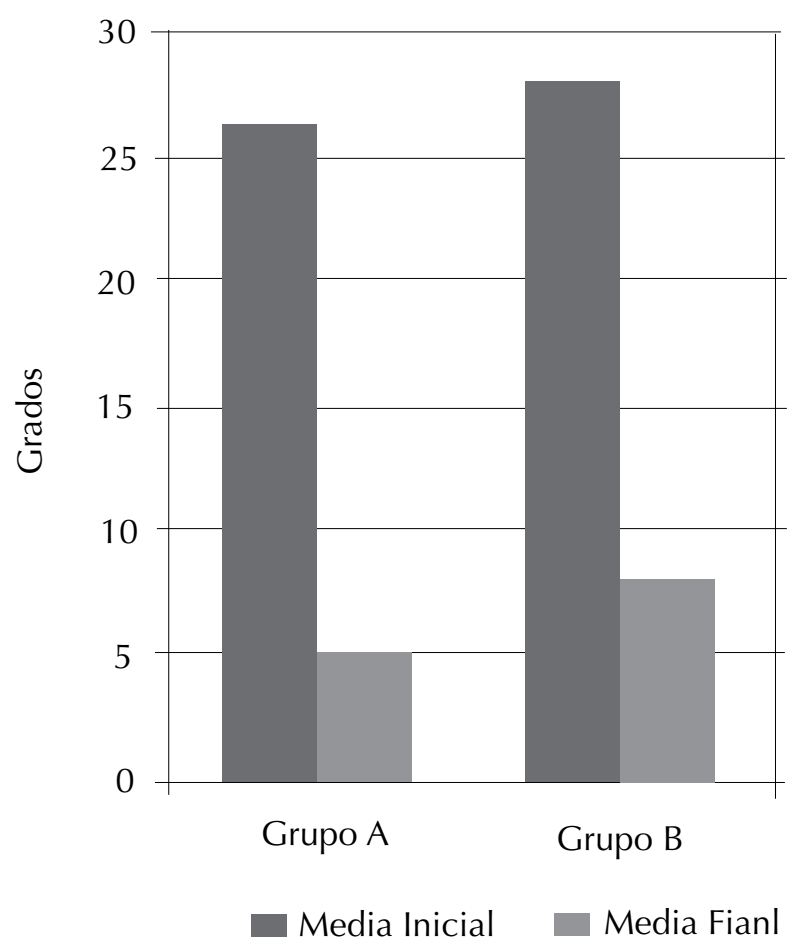

\section{Gráfico 2}

Figura 4. Valores de media de los grados de extensión de la IFP antes y después de la intervención con férulas en el grupo A (férula dinámica) y el grupo $B$ (férula estática).

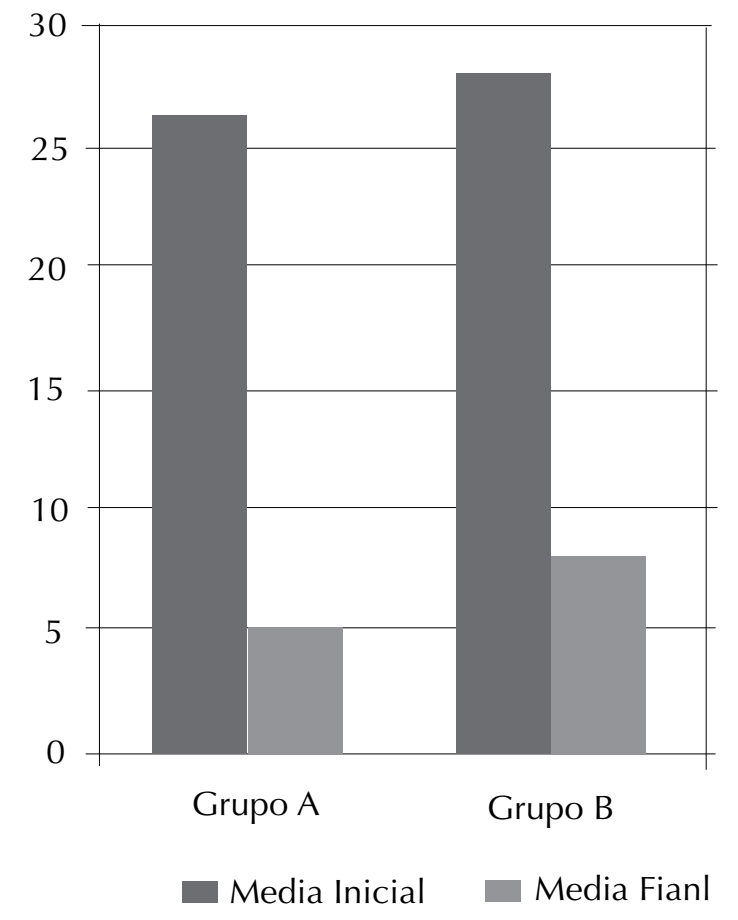

En el caso de los sujetos con una contractura inicial menor de $35^{\circ}$, la media de mejora entre todos los pacientes fue de $20,5^{\circ}$ después de la intervención con la férula. En éstos, la extensión completa del dedo se obtuvo en 2 pacientes (16,6\%). En los otros 10 casos $(83,4 \%)$ quedó un déficit de extensión residual menor de $15^{\circ}$ (Tabla 2).

Si analizamos los datos comparativos entre ambos grupos, se observa que no hubo diferencia estadísticamente significativa entre el grupo que usó férulas dinámicas y el grupo de ferulización estática en la extensión pasiva de la articulación IFP (Tabla 3). 
Tabla 2

Medición del Rango de Movimiento Pasivo en extensión de los 2 grupos con una contractura inicial menor de $35^{\circ}$. Grupos combinados.

\begin{tabular}{|c|c|c|c|c|c|c|}
\hline \multicolumn{3}{|c|}{ Media \pm Desviación estándar } & \multicolumn{4}{c|}{ t-student muestras relacionadas } \\
\hline & Medición inicial & Medición post-tratamiento & Difmed & IC & Valor t & $\mathrm{p}$ \\
\hline $\begin{array}{c}\text { Grados de } \\
\text { extensión IFP }\end{array}$ & $27^{\circ} \pm 4,8$ & $6,50^{\circ} \pm 3,6$ & 20,5 & $\begin{array}{c}{[12,5-} \\
16,8]\end{array}$ & 12,46 & $\mathrm{P}<0,001$ \\
\hline
\end{tabular}

Difmed. Diferencia de Medias

IC. Intervalo de Confianza

Tabla 3

Medición de PROM en extensión de IFP del Grupo A y B tras la intervención.

\begin{tabular}{|c|c|c|c|c|c|}
\hline & \multicolumn{3}{|c|}{ Media \pm Desviación estándar } & \multicolumn{3}{c|}{ t-student muestras independientes } \\
\hline & Grupo A & Grupo B & Difmed & IC & $\mathrm{p}$ \\
\hline Extensión IFP inicial menor 35 $35^{\circ}$ & $5^{\circ} \pm 4,3$ & $8^{\circ} \pm 2,1$ & $3^{\circ}$ & {$[-7,4-1,4]$} & 0,117 \\
\hline \multicolumn{3}{|r}{ Difmed. Diferencia de Medias IC. Intervalo de Confianza } \\
\hline
\end{tabular}

\section{DisCUSIÓN}

Este estudio examinó los efectos de las férulas correctoras en la gestión de la contractura en flexión de la articulación IFP utilizando un programa de rehabilitación combinado de movilización pasiva, ejercicio activo y férulas correctoras.

Después de la intervención con férulas, se observaron mejoras en la corrección de la contractura en flexión de la articulación IFP afectada. Los resultados corroboran los de los estudios previos de Wu (1990) y Li (1999), que muestran que regímenes basados en el uso de férulas correctivas, estáticas o dinámicas, pueden reducir la contractura en flexión de la articulación IFP. Sin embargo, los resultados de este estudio difieren con los análisis biomecánicos realizados por $\mathrm{Li}^{24}$ (1999), que mostró que la férula dinámica de Capener podría generar una mejor fuerza para la corrección óptima de la contractura en flexión de la articulación IFP.

Aunque el análisis de resultados en nuestro caso muestra que no existe diferencia estadísticamente significativa entre el uso de una u otra férula, los datos podrían apoyar el modelo biomecánico dinámico debido a que las observaciones clínicas mostraron que los pacientes en el grupo A recuperaron, en su mayoría, más grados de extensión que los pacientes en el grupo B.
Un estudio realizado en 2002 en dedos reumatoides (Li, 2002) mostró que, aunque el PROM en extensión era similar con el uso de férulas estáticas y dinámicas, existía una diferencia significativa en la flexión resultante de la inmovilización. La mejora en la flexión activa de la articulación IFP fue significativamente mayor en el grupo que usó férulas dinámicas en comparación con el grupo que usó férulas estáticas. Basándonos en el hecho de que la movilización activa de la articulación es útil para corregir la contractura (Colditz JC., 1995) esta mejora observada puede ser explicada por el hecho de que la férula dinámica estimula la flexión activa y la extensión durante el programa con férulas, mientras que los pacientes con férula estática no pueden realizar ambas funciones.

Desarrollar férulas confortables y estéticamente aceptables para el paciente resulta clave a la hora de garantizar la adhesión a su uso. La complejidad de la férula, su comodidad y la interferencia que genera en el estilo de vida y las actividades de la vida diaria o del trabajo son factores que interesan al tiempo de empleo de la férula, repercutiendo en su efectividad final (O'Brien, 2010). En este estudio, los participantes del grupo $\mathrm{B}$ refirieron la necesidad de ajustar la férula tras su aplicación, informando algunos de dificultades en el ajuste y la tensión ejercida. Las férulas dinámicas 
permiten un ajuste continuo y una tensión constante conforme disminuye el ángulo de flexión de la contractura. Las férulas estáticas, por el contrario, requieren de mayores ajustes para que no pierdan efectividad y la fuerza aplicada depende del paciente al colocársela. Sin embargo, la elasticidad de los resortes en las férulas dinámicas debe revisarse con frecuencia ya que, tras periodos prolongados de tensión, gomas y muelles van perdiendo la capacidad de generar suficiente fuerza correctiva en la articulación. Debido a que la remodelación es un proceso biológico que se produce durante largos períodos de tiempo, la frecuencia y la duración de la tensión aplicada, más que la intensidad, resultan claves para maximizar el efecto de las férulas. El uso de una intensa fuerza puede dañar los tejidos, ocasionando una respuesta inflamatoria y la posterior fibrosis. Es imposible, sin embargo, conocer el grado de tensión aplicado en un tejido determinado durante un procedimiento clínico.

Los pacientes de ambos grupos también informaron de una mejoría funcional en las tareas diarias, aunque dicha mejora funcional no fue evaluada en el estudio. Funcionalmente, la articulación IFP es responsable del $85 \%$ del abarcamiento total en la captación de un objeto (Romo R:, Fernández JM., Camacho J., Tarazona P., Quinzaños J., 2010).

Si una articulación IFP está limitada en el movimiento activo, entonces, las actividades que implican mantener una presa para objetos de tamaño medio y pequeño se hacen difíciles. Una vez que la contractura de los tejidos blandos se ha tratado, la articulación está en una buena alineación y el desplazamiento del tendón se produce, desde el punto de vista mecánico, de forma ventajosa, siendo la fuerza de agarre más fuerte.

\section{Limitaciones del estudio}

Este trabajo presenta diversas limitaciones a considerar. La muestra del estudio es pequeña y futuros trabajos deberían ampliarla. No se ha controlado el tiempo transcurrido con rigidez. Además, no se han registrado las horas diarias exactas de uso de la férula, aunque sí se ha marcado un rango límite de uso. Estudios futuros deberían abordar el tiempo y la metodología de aplicación de la férula (intermitente o continua).

En cuanto al diseño del estudio, no se llevó a cabo el ciego del evaluador. Asimismo, sería interesante incluir medidas de resultado funcionales en futuras investiga- ciones que nos permitan correlacionar el incremento en el ROM con mejoras funcionales.

Sería conveniente poder realizar un seguimiento a largo plazo para comprobar si la corrección alcanzada al final del programa de rehabilitación ha perdurado en el tiempo.

\section{Conclusiones}

Según los datos obtenidos en este estudio, un programa combinado de movilización pasiva, ejercicio activo y férulas podría ser eficaz en la corrección de la contractura en flexión de la articulación IFP.

Aunque el uso de férulas para la movilización es una modalidad común usada por terapeutas para mejorar el rango de movimiento, la evidencia disponible para orientar su prescripción por el terapeuta es limitada.

Hasta la fecha, la selección de la férula adecuada para la gestión de la rigidez articular se basa más bien en la experiencia previa de los terapeutas.

Aunque no se observaron diferencias significativas entre la férula dinámica y estática en la recuperación de la extensión de la articulación IFP, la férula dinámica no se asoció con ningún efecto adverso o negativo. Los pacientes no se quejaron de dolor ni fatiga después de usar la férula dinámica. En cambio, aquellos pacientes que llevaron la férula estática manifestaron mayor dificultad en la colocación y ajuste de la misma.

\section{BiBLIOGRAFÍA}

Prosser R. Splinting in the management of proximal interphalangeal joint flexion contracture. J Hand Ther. 1996; 9:378-86.

Frost HM. Skeletal structural adaptations to mechanical usage, 4: mechanical influences on intact fibrous tissues. Anat Rec. 1990; 226:433- 439.

Callahan AD., McEntee P. Splinting proximal interphalangeal joint flexion contractures: a new design. Am J Occup Ther. 1986; 40:408-13.

Brand P. Hand rehabilitation: management by objectives. En: Hunter JM., Schneider LC., Mackin EJ., Callahan AD., editors. Rehabilitation of the Hand. $4^{a}$ ed. St. Louis: Mosby; 1995.

Frank C., Amiel D., Woo SL., Akeson W. Normal ligament properties and ligament healing. Clin Orthop Relat Res. 1985; 196:15-25. 
Peacock EE. Some biomechanical and biophysical aspects of joint stiffness: role of collagen synthesis as opposed to altered molecular boding. Ann Surg. 1983; 164:1-12.

Akeson WH., Amiel D., Woo SL. Immobility effects on synovial joints the pathomechanics of joint contracture. Biorheology. 1980;17:95-110.

Arem AJ., Madden JW. Effects of stress on healing wounds: Intermittent noncyclical tension. J Surg Res. 1976; 20:93-102.

Light KE., Nuzik S., Personius W., Barstrom A. Low-load prolonged stretch vs. high-load brief stretch in treating knee contractures. Phys Ther. 1984; 64:330-3.

Flowers K., LaStayo P. Effect of total end range time on improving passive range of motion. J Hand Ther. 1994;7:150-7.

Wilton J. Hand Splinting: Principles of Design and Fabrication. Londres: WB Saunders; 1997.

Glasgow C., Wilton J., Tooth L. Optimal daily total end range time for contracture: resolution in hand splinting. J Hand Ther. 2003; 16:207-18.

McClure PW., Blackburn LG., Dusold C. The use of splints in the treatment of joint stiffness: biologic rationale and an algorithm for making clinical decisions. Phys Ther. 1994; 74:1101-7.

Flowers K. A proposed decision hierarchy for splinting the stiff joint, with an emphasis on force application parameters. J Hand Ther. 2002; 15: 158-62.

Michlovitz SL., Harris BA., Watkins MP. Therapy Interventions for Improving Joint Range of Motion: A Systematic Review. J Hand Ther. 2004; 17:118-131.

Glasgow C, Fleming J, Tooth L. Which Splint? Dynamic versus static progressive splinting to mobilise stiff joints in the hand. J Hand Ther. 2008;13:104-10.

Fess EE., McCollum M. The influence of splinting on healing tissues. J Hand Ther. 1998; 11:157-161.

Abbiati G., Delaria G., Saporiti E., Petrolati M., Tremolada C. The treatment of chronic flexion contractures of the proximal interphalangeal joint. J Hand Surg Br. 1995; 20: 385-9.

Freeland AE., Hardy MA., Singletary S. Rehabilitation for Proximal Phalangeal Fractures. J Hand Ther. 2003; 16:129-142.

Glasgow C., Tooth LR., Fleming J. Mobilizing the Stiff Hand: Combining Theory and Evidence to Improve Clinical Outcomes. J Hand Ther. 2010; 23:392-401.

Fairleigh A., Hacking S. Post-operative metacarpophalangeal arthroplasty dynamic splint for patients with rheumatoid arthritis. Can J Occup Ther. 1988; 55: 141-6.

Palchik NS., Mitchell DM., Gilbert NL., Schulz AJ., Dedrick RF., PaleIla TD. Nonsurgical management of the boutonniere deformity. Arthritis Care Res. 1990; 3:227-32.

Wu SH: A belly gutter splint for proximal interphalangeal joint flexion contracture. Amer Jour Occup Therapy. 1990, 45:939-943.

Li C. Force analysis of the Belly Gutter and Capener splints. J Hand Ther. 1999; 12:337-343.
Fess EE. Force magnitude of commercial spring-coil and sring-wire splint designed to extend the proximal interphalangeal joint. J Hand Ther. 1988; 1:86-90.

Fess EE., Philips CA. Hand splinting: principles and methods. $2^{\text {a }}$ ed. St. Louis: Mosby; 1987.

Colditz J. Efficient Mechanics of PIP Mobilisation Splinting. J Hand Ther. 2000; 5:65-71.

Fess EE. Hand and upper extremity splinting: principles \& methods. $3^{\text {a }}$ ed. St. Louis: Mosby; 2004.

Hunter JM., Mackin EJ., Callahan, AD. Rehabilitation of the Hand. $4^{\mathrm{a}}$ ed. St. Louis: Mosby; 1995.

Li-Tsang CW., Hung LK., Mak AF. The effect of corrective splinting on flexion contracture of rheumatoid fingers. J Hand Ther. 2002; 15:185-91. 